\title{
Evaluation of Neutrophil-to-Lymphocyte Ratio, Platelet-to-Lymphocyte Ratio, and C-Reactive Protein in Tension-Type Headache Patients
}

\author{
Hasan Hüseyin Özdemir ${ }^{1, \odot ~ A h m e t ~ D o ̈ n d e r ², \odot ~}$ \\ ${ }^{1}$ Department of Neurology, A Hospital, İstanbul, Turkey \\ ${ }^{2}$ Department of Medical Laboratory, Vocational School of Health \\ Services, Mardin Artuklu University, Mardin, Turkey
}

Address for correspondence Ahmet Dönder, PhD, Department of Medical Laboratory, Vocational School of Health Services, Mardin Artuklu University, Mardin 47200, Turkey (e-mail: ahmetdundar83@hotmail.com).

\begin{abstract}
Keywords

- neutrophil-tolymphocyte ratio

- platelet-to-lymphocyte ratio

- tension-type headache

Objectives A tension headache is the most common type of headache, and its causes are multifactorial. A relationship has been shown between migraine headaches and neutrophil-to-lymphocyte ratio (NLR), platelet-to-lymphocyte ratio (PLR), and C-reactive protein (CRP). In this study, we investigated the NLR, PLR, and serum CRP levels in frequent episodic tension-type headache (FETTH) and chronic tension-type headache (CTTH) patients.

Materials and Methods This retrospective study included 64 patients with FETTH, 80 patients with $\mathrm{CTTH}$, and 60 healthy controls who were followed up in the neurology clinic. Hematological parameters were compared between the patient and control groups.

Results In CTTH patients, platelets, NLR, PLR, and CRP values were statistically higher than in FETTH patients and patients in the control group. In FETTH patients, the PLR value was higher than in patients in the control group, but there was no statistically significant difference in NLR and CRP values between FETTH patients and patients in the control group. Also, there was no correlation between these values and age and gender.

Conclusion Increase platelet count might have an effect on tension-type headache pathophysiology. Systemic inflammation parameters were shown to be significantly higher in CTTH patients. More comprehensive studies are needed to evaluate the effect of systemic inflammation on the chronicity of tension headaches.
\end{abstract}

\section{Introduction}

Tension-type headache (TTH) is a common primary headache type whose causes are multifactorial. Genetic and environmental factors play a critical role. ${ }^{1}$ There are episodic and chronic forms of TTH, and there are multiple distinctions between forms. Episodic-type headaches often evolve into chronic-type headaches over time. ${ }^{2}$ Previous studies have reported that peripheral and central sensitization mechanisms may play a role in the chronification of headaches. ${ }^{3}$ The effects of local and systemic inflammation on tension headaches are not clearly known.

Recent studies have demonstrated that neutrophilto-lymphocyte ratio (NLR) and platelet-to-lymphocyte ratio published online June 15, 2021
DOI https://doi.org/

$10.1055 / \mathrm{s}-0041-1730124$ ISSN 0976-3147

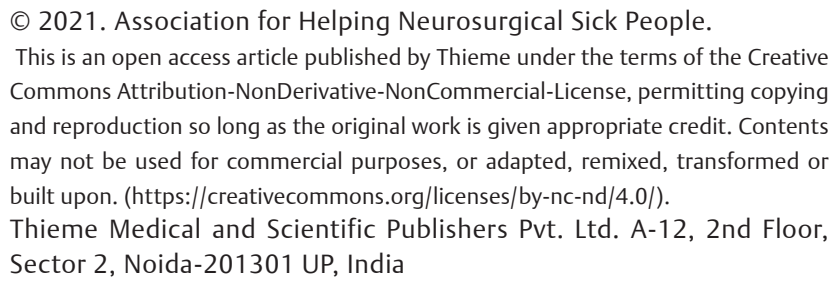

(c) 2021. Association for Helping Neurosurgical Sick People.

This is an open access article published by Thieme under the terms of the Creative Commons Attribution-NonDerivative-NonCommercial-License, permitting copying and reproduction so long as the original work is given appropriate credit. Contents may not be used for commercial purposes, or adapted, remixed, transformed or built upon. (https://creativecommons.org/licenses/by-nc-nd/4.0/). Thieme Medical and Scientific Publishers Pvt. Ltd. A-12, 2nd Floor, Sector 2, Noida-201301 UP, India 
(PLR) are important indicators of systemic inflammation. It has also been shown to have effects on inflammation in neurological diseases such as Guillain-Barre's syndrome and convulsive status epilepticus. ${ }^{4,5}$ NLR and PLR are noninvasive evaluation markers that can easily be determined from peripheral blood samples. It has been stated that platelets may have an effect on the pathophysiology of tension headaches, and platelet serotonin levels in TTH may be related to pain. ${ }^{6}$

C-reactive protein (CRP), which is an acute phase reactant associated with acute and chronic inflammation, plays a role in pathological processes, and studies have found that increased CRP levels are involved in neurological diseases. ${ }^{7}$ In particular, CRP has been associated with migraine headaches. ${ }^{8}$ It has been noted that platelets play a role in inflammatory processes and immune responses. ${ }^{9} \mathrm{~A}$ study of migraine patients and the biological functions of platelets showed that platelets can play an active role in migraine headache etiology. ${ }^{10}$ However, the effect of platelets on the pathophysiology of tension headache disease is not fully known. Also, NLR, PLR, and CRP have not been previously evaluated in TTH. This study aimed to investigate the role of platelets, NLR, PLR, and CRP in frequent episodic tension-type headache (FETTH) and chronic tension-type headache (CTTH) patients.

\section{Materials and Methods}

In this study, 144 patients who had a diagnosis of TTH between January 2017 and August 2020 in the Department of Neurology, "A" Hospital were retrospectively evaluated. A control group of 60 healthy volunteers who were admitted to the checkup in the polyclinic was included. Demographic features, including age and sex, clinical features, frequency of pain, drugs being used, and medical history were assessed. The diagnosis of TTH was based on the International Classification of Headache Disorders-III diagnostic criteria. ${ }^{11}$ The patients were divided into FETTH and CTTH groups according to their clinical characteristics. Duration of disease was determined for the patient groups.

All of the headache patients underwent physical, neurological, and fundus examinations. Kidney and liver function test levels and electrolyte levels were measured, and magnetic resonance imaging (MRI) of the brain was performed. Patients with intracranial pathology in the MRI of the brain were not included in the study. Blood samples were collected when the patient presented to the neurology policlinic. The exclusion criteria included a history of other neurologic diseases, such as migraine, pregnancy, autoimmune diseases, diabetes mellitus, malignant hypertension, trauma, malignancy, thyroid diseases, liver and kidney dysfunction, and local or systemic infection. In addition, patients who had a headache at the time of examination or had used analgesics in the preceding 72 hours were excluded.

\section{Hematological and Biochemical Analyses}

Serum CRP level was measured with a Beckman Coulter CX9 (Beckman Coulter, Inc., Brea, California, United States) chemistry analyzer. Hematologic indices were measured using an automated hematology analyzer system (Abbott Cell-Dyn 3700; Abbott Laboratory, Abbott Park, Illinois, United States). All the subsequent analyses were based on absolute cell counts. The baseline NLR was calculated by dividing the neutrophil count by the lymphocyte count, and the PLR was calculated by dividing the platelet count by the lymphocyte count.

\section{Statistical Analyses}

Statistical analyses were performed using the Statistical Package for the Social Sciences 21.0 for Windows software program (SPSS Inc., Chicago, United States). Continuous data are presented as mean \pm standard deviation. The KruskalWallis' test was used to compare more than two groups. In comparison to the Kruskal-Wallis' test, Bonferroni correction was performed, and the Mann-Whitney's $U$ test was used to compare the two groups. The cutoff values and corresponding sensitivity and specificity values for the prediction between the CTTH group and the FETTH group based on serum PLR, NLR, and CRP were estimated by receiver operating characteristic (ROC) curve analysis. A result was accepted as statistically significant with a $p$-value $<0.05$.

\section{Results}

Sixty-four patients with FETTH, 80 patients with CTTH, and 60 healthy controls were enrolled in this study. The mean age of the FETTH patients was $34.62 \pm 6.74$ years, and the mean disease duration of the FETTH patients was $9.16 \pm$ 6.69 months. The mean age of the CTTH patients was 34.67 \pm 6.41 years, and the mean disease duration of the CTTH patients was $12.20 \pm 4.03$ months. The mean age of the control group was $35.60 \pm 10.14$ years. There was no statistically significant difference between the groups in terms of sex and age.

While the mean white blood cell (WBC) count in the FETTH patients was found to be $7.97 \pm 0.75$, it was $7.95 \pm$ 2.21 in the CTTH patients and $8.38 \pm 0.69$ in the control group. When the WBC count was compared between the two headache groups, it was found to be statistically significantly higher in the CTTH patients $(p<0.001)$. The platelet count in the CTTH patient group was higher than in the other groups. Furthermore, the platelet counts in both the FETTH and CTTH patients were higher than in the control group. The NLR, PLR, and CRP values in the CTTH patients were statistically higher than in the FETTH patients and control groups. There was no correlation between age, disease duration, NLR, PLR, and CRP values between the two patient groups. The hematological parameters of the FETTH, CTTH, and control groups are presented in - Table 1 .

A cutoff NLR of 2.58 predicted the difference between the CTTH group and the FETTH group, with $19.4 \%$ sensitivity and $93.3 \%$ specificity (ROC area under the curve [AUC] of 0.61 and $95 \%$ confidence interval $[\mathrm{CI}]$ of $0.500-0.734)$. A cutoff PLR of 236.6 predicted the difference between the CTTH group and the FETTH group, with 9.7\% sensitivity and 96.7\% specificity (ROC AUC of 0.66 and $95 \%$ CI of $0.551-0.782$ ). 
Table 1 Comparison of demographic and hematological parameters of the frequently episodic group, chronic tension-type headache group, and control group

\begin{tabular}{|c|c|c|c|c|c|}
\hline \multirow[t]{2}{*}{ Parameter } & $\begin{array}{l}\text { Control } \\
\text { group }\end{array}$ & $\begin{array}{l}\text { Frequently } \\
\text { episodic group }\end{array}$ & $\begin{array}{l}\text { Chronic tension- } \\
\text { type headache } \\
\text { group }\end{array}$ & \multirow[t]{2}{*}{$p$-Value } & \multirow[t]{2}{*}{$95 \% \mathrm{Cl}$} \\
\hline & $(n=60)$ & $(n=64)$ & $(n=80)$ & & \\
\hline Age & $35.60 \pm 10.14$ & $34.62 \pm 6.74$ & $34.67 \pm 6.41$ & 0.993 & $0.385-0.693$ \\
\hline $\mathrm{WBC}\left(\times 10^{3} / \mathrm{mL}\right)$ & $7.94 \pm 0.82$ & $7.9 \pm 0.75$ & $8.38 \pm 0.69^{b}$ & 0.028 & $0.464-0.716$ \\
\hline Neutrophil $\left(\times 10^{3} / \mathrm{mL}\right)$ & $3.80 \pm 0.70$ & $3.77 \pm 0.64$ & $3.96 \pm 0.64$ & 0.321 & $0.396-0.645$ \\
\hline Lymphocytes $\left(\times 10^{3} / \mathrm{mL}\right)$ & $2.27 \pm 0.71$ & $2.21 \pm 0.74$ & $1.90 \pm 0.46$ & 0.100 & $0.285-0.531$ \\
\hline Platelets $\left(\times 10^{3} / \mathrm{mL}\right)$ & $\begin{array}{l}282.18 \pm \\
52.70\end{array}$ & $286.26 \pm 60.92$ & $333.40 \pm 49.23^{b}$ & $<0.001$ & $0.530-0.763$ \\
\hline CRP $(\mathrm{mg} / \mathrm{dL})$ & $0.27 \pm 0.12$ & $0.28 \pm 0.12$ & $0.39 \pm 0.08^{b}$ & $<0.001$ & $0.528-0.768$ \\
\hline NLR & $1.80 \pm 0.61$ & $1.88 \pm 0.69$ & $2.21 \pm 0.70^{\mathrm{b}}$ & 0.027 & $0.500-0.734$ \\
\hline PLR & $\begin{array}{l}136.12 \pm \\
50.31\end{array}$ & $144.31 \pm 57.36^{a}$ & $185.90 \pm 58.52^{c}$ & $<0.001$ & $0.551-0.782$ \\
\hline
\end{tabular}

Abbreviations: Cl, confidence interval; CRP, C-reactive protein; NLR, neutrophil-to-lymphocyte ratio; PLR, platelet-to-lymphocyte ratio; SD, standard deviation; WBC, white blood cell.

Note: Data are expressed as (mean \pm SD).

${ }^{a} p<0.01$ the degree of significance of comparison between the patient and control groups.

${ }^{b} p<0.05$, the degree of significance of comparison between the patient and control groups.

${ }^{c} p<0.001$, the degree of significance of comparison between the patient and control groups.

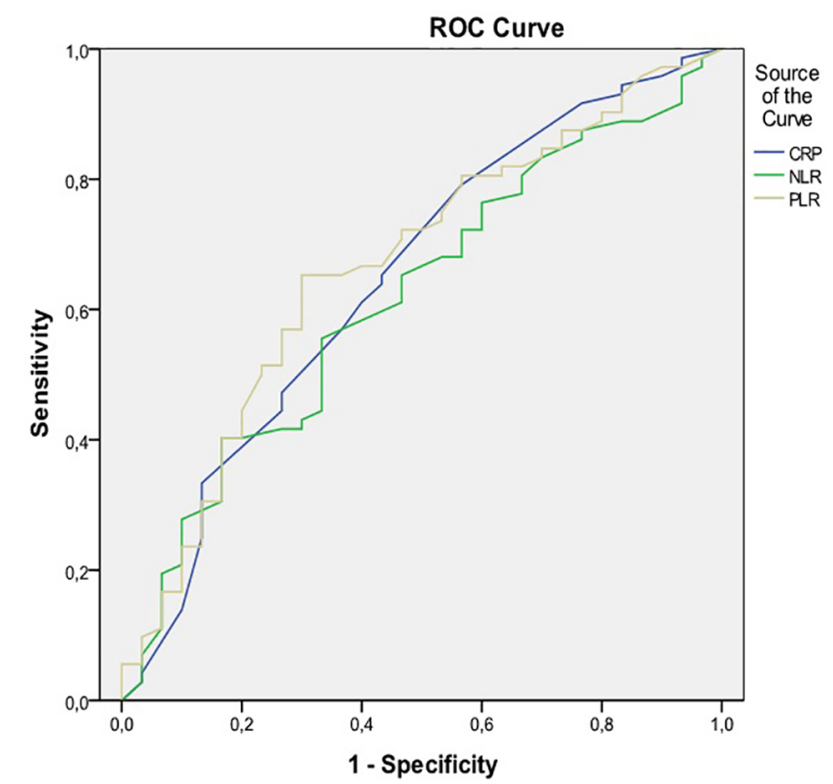

Fig. 1 The ROC curve analysis of NLR, PLR, and CRP for prediction between the frequently episodic group and chronic tension-type headache group. CRP, C-reactive protein; NLR, neutrophil-to-lymphocyte ratio; PLR, platelet-to-lymphocyte ratio; ROC, receiving operating characteristic.

A cutoff CRP of 0.42 predicted the difference between the CTTH group and the FETTH group, with $33.3 \%$ sensitivity and $86.7 \%$ specificity (ROC AUC of 0.64 and 95\% CI of 0.528-0.768 $p>0.05$; - Fig. 1).

\section{Discussion}

In this study, we first determined that platelet counts and NLR, PLR, and CRP values were statistically higher in the
CTTH patients than in the other two groups. There was no difference in NLR and CRP values between the TTH patients and in the control group. We found that the platelet counts were significantly higher in the patient groups than in the control group. There was no correlation between age, frequency and duration of pain, and inflammatory markers in the patient groups. This study shows that increased platelet counts may play a role in the pathophysiology of TTH, and systemic inflammation may be a factor that affects the chronicity of tension headaches.

Peripheral mechanisms are predominant in FETTH, but central mechanisms are involved in CTTH. Recent studies have shown that pericranial myofascial pain sensitivity is increased in TTH and may play an important role in the pathophysiology of these headaches. ${ }^{12}$ On the contrary, sensitization of second-order neurons at the level of the spinal dorsal horn, trigeminal nucleus, or supraspinal neurons, as well as decreased descending inhibition from supraspinal structures may be involved in the pathophysiology of CTTH. ${ }^{13}$ Also, drug overuse and substance abuse suggest that a genetic factor is involved in the process of headache chronification. ${ }^{14}$ There are limited studies evaluating tension headache and peripheral inflammation. Domingues et al showed that the level of interleukin (IL)-8 and monocyte chemoattractant protein-1 increased in TTH types, and they suggested that proinflammatory mechanisms may participate in TTH pathophysiology. ${ }^{15}$ NLR is one of the important indicators of inflammation that is associated with many diseases. Studies have been conducted, especially in patients with migraine headaches during attacks, and found that NLR and PLR were higher in the patients compared with the controls. ${ }^{16}$ A study by Eryigit et al identified higher NLR levels in patients with subarachnoid hemorrhage compared with patients with migraine or other headache syndromes. ${ }^{17}$ In 
our study, no statistically significant change was observed in the neutrophil and lymphocyte counts in the patient groups. We only found NLR levels to be significantly higher in CTTH patients. No difference was observed between the FETTH patients and the control group.

The importance of platelets in headaches has been studied for many years. In migraine cases, essential thrombocythemia supports the fact that increased platelets can lead to headaches. ${ }^{10}$ There are limited studies evaluating the relationship between platelets and TTH. Leira et al showed that platelet-rich plasma serotonin levels were higher in patients with TTH. ${ }^{6}$ Blood serotonin levels reflect intraplatelet serotonin because most blood serotonin is stored inside platelets. ${ }^{18,19}$ Also, increased platelet levels are an important indicator of an inflammatory response. ${ }^{20}$ Ulusoy showed that mean platelet volume (MPV) and MPV-to-platelet ratio increased in a statistically significant manner in migraine patients, but MPV and MPV-to-platelet ratio had similar levels between FETTH patients and the healthy population. ${ }^{21}$ In this study, the platelet counts in patients with TTH were significantly higher. ${ }^{21}$ Benedick et al found that platelet counts in children with TTH were higher than in migraine patients. ${ }^{22}$ In our study, platelet counts were higher in both FETTH and CTTH patients. We also found that the PLR value was higher in patients than in the control group. The higher platelet counts may have been affected by the serotonin levels, or it may be an indicator of the inflammatory response in the chronicity of the disease. Detection of increase in the platelets in the regular follow-up of TTH patients may indicate that the disease may have become chronic. Large-scale studies are needed to support these findings.

The relationship between CRP and migraine has been evaluated in many studies. ${ }^{23} \mathrm{CRP}$ is an acute phase protein that is produced predominantly by the action of cytokines, such as IL- 6 and tumor necrosis factor- $\alpha .{ }^{10}$ CRP levels were found to be higher in patients admitted with the complaint of migraine attacks compared with controls. ${ }^{24}$ However, the relationship between CRP and CTTH is not clearly known. Bougea et al found no significant differences in CRP, IL-1 $\beta$, and IL-6 levels between migraine patients and TTH patients. ${ }^{25}$ In our literature review, we first found high CRP levels in CTTH patients. Also, the sensitivity of our NLR, PLR, and CRP tests was found to be very low due to the low area below the ROC curve, but the likelihood ratios of NLR, PLR, and CRP were good.

The principal limitation of this study is that platelet and serotonin levels were not evaluated together. These findings may uncover the importance of platelets in TTH pathophysiology.

\section{Conclusion}

In conclusion, increased platelet counts may have an effect on TTH pathophysiology. In addition, systemic inflammation parameters, such as NLR, PLR, and CRP, increased in the chronic phase of the disease. Therefore, we think that more comprehensive studies are needed to evaluate the effect of platelets on TTH and systemic inflammation in the chronicity of TTH.
Conflict of Interest

None declared.

\section{References}

1 Scripter C. Headache: tension-type headache. FP Essent 2018; 473:17-20

2 Kropp P, Egli G, Sándor PS. Tension-type headache introduction and diagnostic criteria. Handb Clin Neurol 2010;97:355-358

3 Bendtsen L, Fernández-de-la-Peñas C. The role of muscles in tension-type headache. Curr Pain Headache Rep 2011;15(6):451-458

4 Ozdemir HH. Analysis of the albumin level, neutrophil-lymphocyte ratio, and platelet-lymphocyte ratio in Guillain-Barré syndrome. Arq Neuropsiquiatr 2016; 74(9):718-722

5 Özdemir HH, Akil E, Acar A, et al. Changes in serum albumin levels and neutrophil-lymphocyte ratio in patients with convulsive status epilepticus. Int J Neurosci 2017;127(5):417-420

6 Leira R, Castillo J, Martinez F, Prieto JM, Noya M. Platelet-rich plasma serotonin levels in tension-type headache and depression. Cephalalgia 1993;13(5):346-348

7 Yazar T, Yazar HO. Evaluation of C-reactive protein/albumin ratio according to stage in patients with idiopathic Parkinson disease. Turk J Neurol 2019;25:123-128

8 Hagen K, Stovner LJ, Nilsen KB, Kristoffersen ES, Winsvold BS. The impact of C-reactive protein levels on headache frequency in the HUNT study 2006-2008. BMC Neurol 2019;19(1):229

9 Gasparyan AY, Ayvazyan L, Mikhailidis DP, Kitas GD. Mean platelet volume: a link between thrombosis and inflammation? Curr Pharm Des 2011;17(1):47-58

10 Danese E, Montagnana M, Lippi G. Platelets and migraine. Thromb Res 2014;134(1):17-22

11 Headache Classification Committee of the International Headache Society (IHS). The International Classification of Headache Disorders.3rd edition.Cephalalgia 2018;38(1):1-211

12 Chen Y. Advances in the pathophysiology of tension-type headache: from stress to central sensitization. Curr Pain Headache Rep 2009;13(6):484-494

13 AshinaS, Bendtsen L, Ashina M. Pathophysiology of tension-type headache. Curr Pain Headache Rep 2005;9(6):415-422

14 Cevoli S, Sancisi E, Grimaldi D, et al. Family history for chronic headache and drug overuse as a risk factor for headache chronification. Headache 2009;49(3):412-418

15 Domingues RB, Duarte H, Rocha NP, Teixeira AL. Increased serum levels of interleukin-8 in patients with tension-type headache. Cephalalgia 2015;35(9):801-806

16 Yazar HO, Yazar T, Aygün A, Kaygisiz Ş, Kirbaş D. Evaluation of simple inflammatory blood parameters in patients with migraine. Ir J Med Sci 2020;189(2):677-683

17 Eryigit U, Altunayoglu Cakmak V, Sahin A, et al. The diagnostic value of the neutrophil-lymphocyte ratio in distinguishing between subarachnoid hemorrhage and migraine. Am J Emerg Med 2017;35(9):1276-1280

18 Beikmann BS, Tomlinson ID, Rosenthal SJ, Andrews AM. Serotonin uptake is largely mediated by platelets versus lymphocytes in peripheral blood cells. ACS Chem Neurosci 2013;4(1):161-170

19 Xiao R, Beck O, Hjemdahl P. On the accurate measurement of serotonin in whole blood. Scand J Clin Lab Invest 1998;58(6):505-510

20 Stokes KY, Granger DN. Platelets: a critical link between inflammation and microvascular dysfunction. J Physiol 2012; 590(5):1023-1034

21 Ulusoy EK. Use of MPV and MPV/PLT ratio in the differentiation of migraine and tension-type headache. Acta Haematol Pol 2018;49(4):15-19 
22 Benedick A, Zeharia A, Markus TE. Comparison of thrombocyte count between pediatric patients with migraine or tension-type headache: a retrospective cohort study. J Child Neurol 2019;34(13):824-829

23 VanmolkotFH, de HoonJN.Increased C-reactive protein in young adult patients with migraine. Cephalalgia 2007;27(7):843-846

24 Cerman AA, Karabay EA, Altunay IK. The evaluation of the relationship between the response of systemic treatment with C-reactive protein and neutrophil lymphocyte ratio in psoriasis patients: a preliminary study. Turk Klin J Dermatol 2016;26:71-75

25 Bougea A, Spantideas N, Galanis P, et al. Salivary inflammatory markers in tension type headache and migraine: the SalHead cohort study. Neurol Sci 2020;41(4):877-884 